\title{
Non-destructive investigation of polymer-metal bonds using a VibroMap1000 holographic vibrometer
}

\author{
Zawadzki Pawel ${ }^{1,2^{*}}$, Meijer Frans ${ }^{2}$, Stachowska Ewa ${ }^{2}$ and Legutko Stanisław ${ }^{1}$ \\ ${ }^{1}$ Chair of Management and Production Engineering, Faculty of Mechanical Engineering and \\ Management, Poznan University of Technology, PL-60-965 Poznan, Poland. \\ ${ }^{2}$ Department of Metrology and Measurement Systems, Faculty of Mechanical Engineering and \\ Management, Poznan University of Technology, PL-60-965 Poznan, Poland.
}

\begin{abstract}
We developed a method to test polymer-metal bonds using a VibroMap1000 holographic vibrometer. The set-up and the data processing system allow non-destructive testing of polymer-metal bonds for samples of a specific size and structure. Frequency scanning from 200 $\mathrm{Hz}-20 \mathrm{kHz}$ provides characteristics of the sample. Because the method is non-destructive the sample can be studied afterwards with other methods too. These combined with the measurements of the holographic vibrometer, can create a complete and accurate assessment of the state of polymer-metal bonds. We present some preliminary results.
\end{abstract}

\section{Introduction}

The growing use of polymers requires searching for various solutions to combine it with metal and ceramic materials. Parts made by a metal-polymer connection are widely used in the automotive, aerospace and medical industries [1]. These elements are characterized by a combination of metal strength and ductility with physical and chemical resistance and low polymer weight.

Methods based on adhesion are widely used in connection technology. Particular attention should be paid to the Outsert technique [2]. The polymer is injected into simple metal parts placed in the matrix. This process enables the formation of complex components of low weight and high strength [2]. In adhesive connections, the quality of the connection depends on many factors, including preparation of the metal surface, the nature of polymer shrinkage and the possibility of stress formation. The most popular methods of testing these connections are: analysis of the stretching or compression or cracking resistance carried out in various types of environments and temperatures. However, these methods damage the sample eliminating the possibility of further studies [3].

Non-destructive testing enables to obtain information about the physical condition of an element, without causing changes in its functional properties. Elements can be monitored during production and exploitation. In studies of polymers and composites the following methods are used: ultrasound defectoscopy [4], radiography [5], shearography [6],

\footnotetext{
${ }^{*}$ Corresponding author: pawel.zawadzki@put.poznan.pl
} 
penetration analysis, vibrometry including digital holographic $[7,8,9,10]$, used in this work. Digital holographic vibrometry used in this work allow for non-destructive testing on metal-polymer samples. The results presented in the following fragments of the paper confirm the correctness and usefulness of its application.

\section{Experimental work}

\subsection{Laser vibrometer}

A laser vibrometer consist of a laser light source, a Michelson interferometer and a detection sys-tem with a CCD camera. The object to test is excited to vibration using a piezoelectric transducer, function generator and signal amplifier. The laser beam directed to the sample is reflected from the surface of the object, which results in deformation of the wave front. This beam interferes with the reference beam and create a hologram. The deformation of the object can be obtained in 3D when reconstructing the object beam from the hologram. The vibrometer was equipped with a frequency doubled solid-state $\mathrm{Nd}$ : YAG laser emitting a wavelength of $532 \mathrm{~nm}$.

\subsection{Experimental setup}

We used an experimental setup consisting of: an Optonor VibroMap 1000 holographic vibrometer, a function generator Tektronix AFG3022C, a signal amplifier Physics Instruments E0501, an antivibration table with a steel top and a PC to control the excitation and image recording. The software controlling the laser vibrometer was Optonors VibroMap_B4 - based on MATLAB. A simple piezoelectric transducer connected to the signal amplifier was used to exite the sample to vibrate. During the design of the test setup, several versions of the measurement system were tried. We finally decided to use a vertical mounting system of the sample with the piezoelectric transducer directly fixed to the sample.

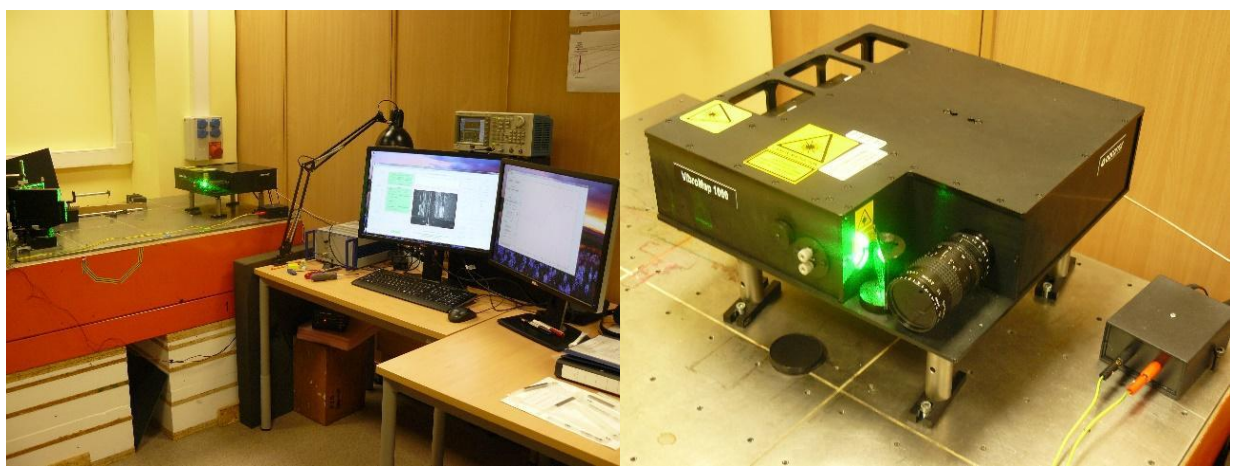

Fig. 1. General view of the holographic vibrometer setup (on the left) and Vibromap unit (on the right).

Various methods of fixing the sample were tested, enabling vibration excitation and simultaneous recording the sample on both sides of the polymer-metal laminate. The piezotransducer was in direct contact with the polymer surface of the sample at a (fixed) distance of $15 \mathrm{~mm}$ from the end. To maximize the freedom of vibration in the sample, it was mounted on both edges in V-shaped grooves. Simultaneous observation of vibrations 
from the side of metal and polymer was possible by a system of flat mirrors. The mounting system is shown in figure 2 .

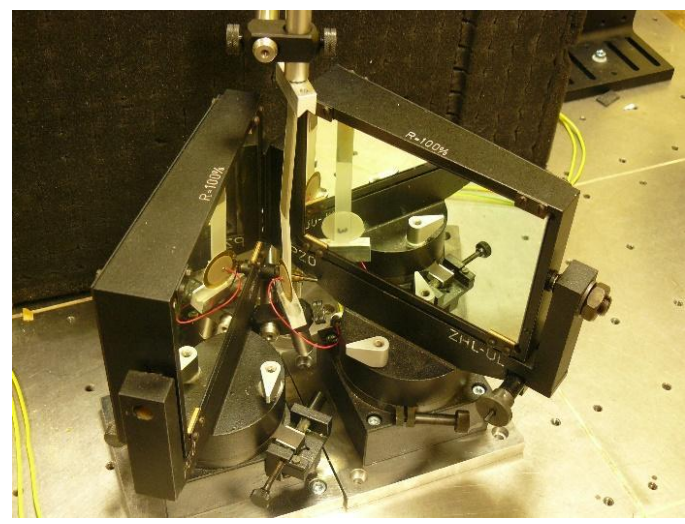

Fig. 2. Top view of the mounting and excitation system of the sample, vertically mounted in the centre of the mirrors with a circular piezoelectric transducer at the bottom.

The test samples were a combination of a polymer and a metal part. The polymer material was polypropylene PP HP500N / TE10 and the metal was DC04 steel. The samples consisted of a strip with dimensions of $150 \times 10 \times 26 \mathrm{~mm} 3$ or $150 \times 10 \times 21 \mathrm{~mm} 3$ (length $\mathrm{x}$ width $\mathrm{x}$ thickness), consisting of a plate of a polymer with dimensions of $150 \mathrm{x}$ $10 \times 25 \mathrm{~mm} 3$ or $150 \times 10 \times 20 \mathrm{~mm} 3$ and a steel one with dimensions of $76 \times 10 \times 1 \mathrm{~mm} 3$. A typical sample is shown in figure 3. Metal and polymer parts were bonded using Outsert technique [2] mentioned in this work introduction.

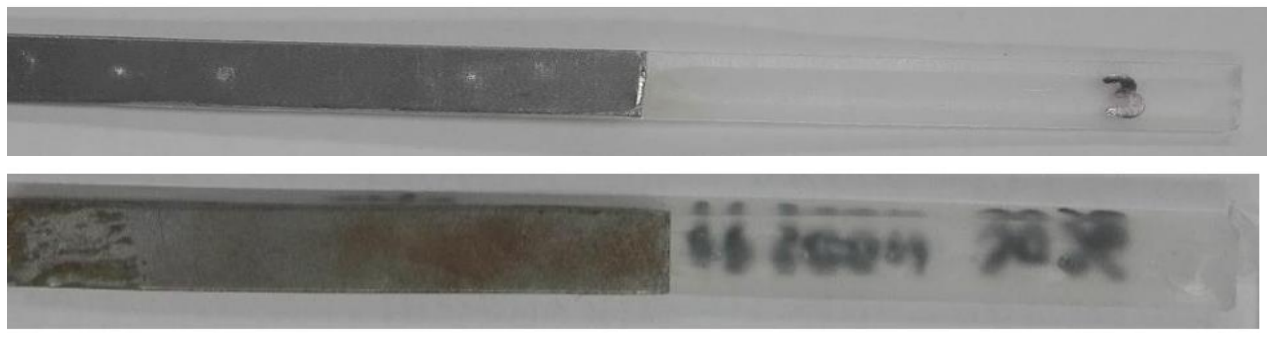

Fig. 3. View of samples with a metal strip on the right side.

\subsection{Experimental setup}

We tested with the following parameters: vibration amplitude $5000 \mathrm{mV}$ p-p multiplied by the amplifier to $500 \mathrm{~V}$ and excitation frequency range: $150-25000 \mathrm{~Hz}$ with $10 \mathrm{~Hz}$ steps. The frequency scan was repeated three times for each sample. Due to the possible application of polymers combined with metals, particular attention was paid to observing vibrations at lower frequencies: $150-5000 \mathrm{~Hz}$.

\section{Results}

The Vibromap program can provide a movie of the deflection along a chosen line. Thirty pictures can be stored, corresponding to one vibration period. These pictures were digitized 
to obtain the deflection along the central line chosen in steps of 1/30 period. We developed a program in the programming language " $R$ " [11], which offers powerful statistical and visualization possibilities and is open source, running on a desktop computer with GNU/Linux. Eventual torsion of the sample is left out of consideration with this method of data handling.

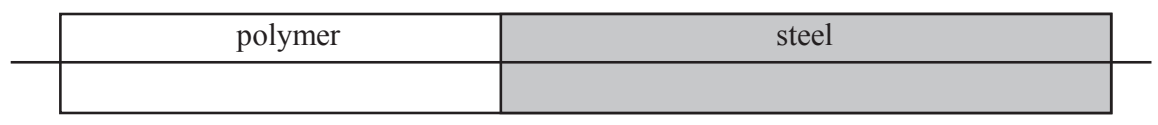

Fig. 4. View of a sample from the top, the dotted line shows the line of observation.

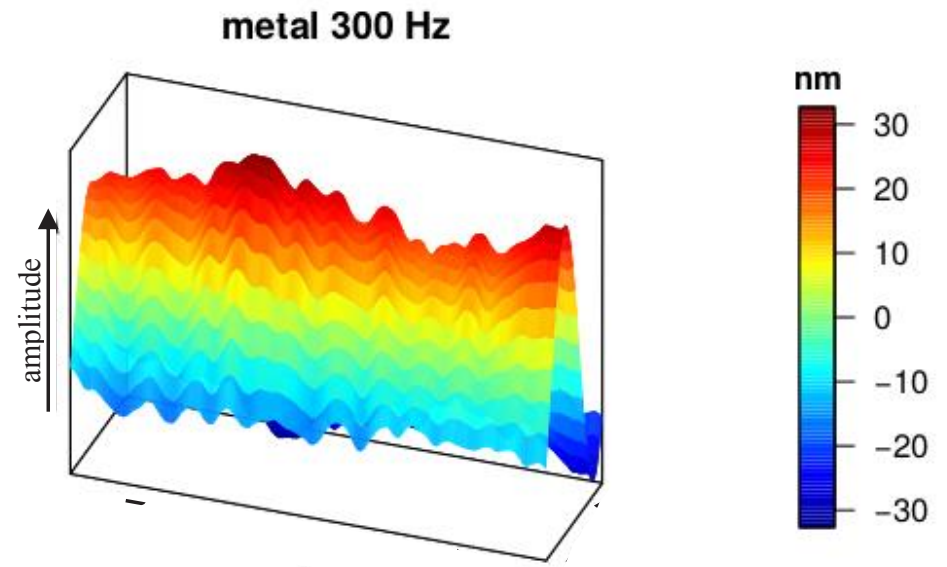

position of the sample

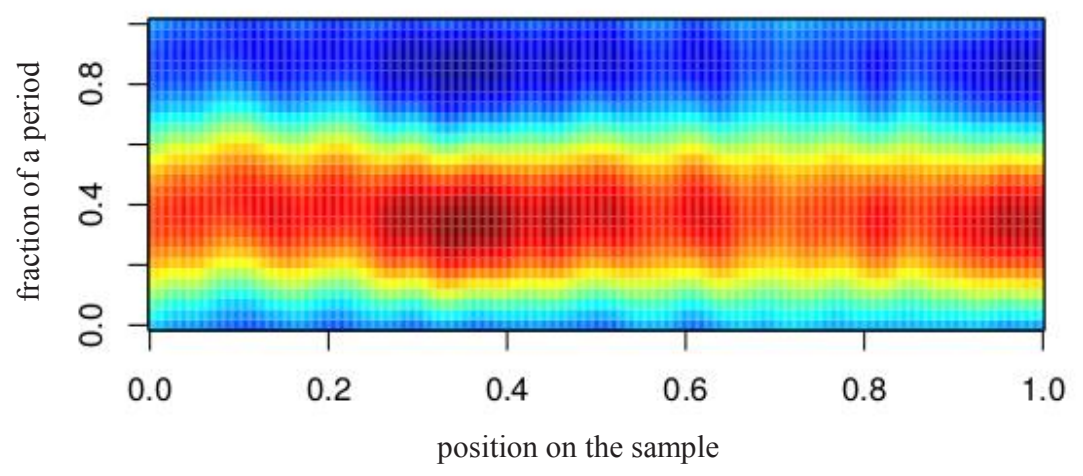

Fig. 5. Deflection along a central line of the sample during one period at $300 \mathrm{~Hz}$.

Figure 5 shows the change in deflection of the sample during one period of time. For every sample observations were made along the line passing through the centre of the sample (see figure 4). In the graph above, the maximum at certain positions and times are noticeable - around 0.3-0.4 and 1.0. 
metal $300 \mathrm{~Hz}$

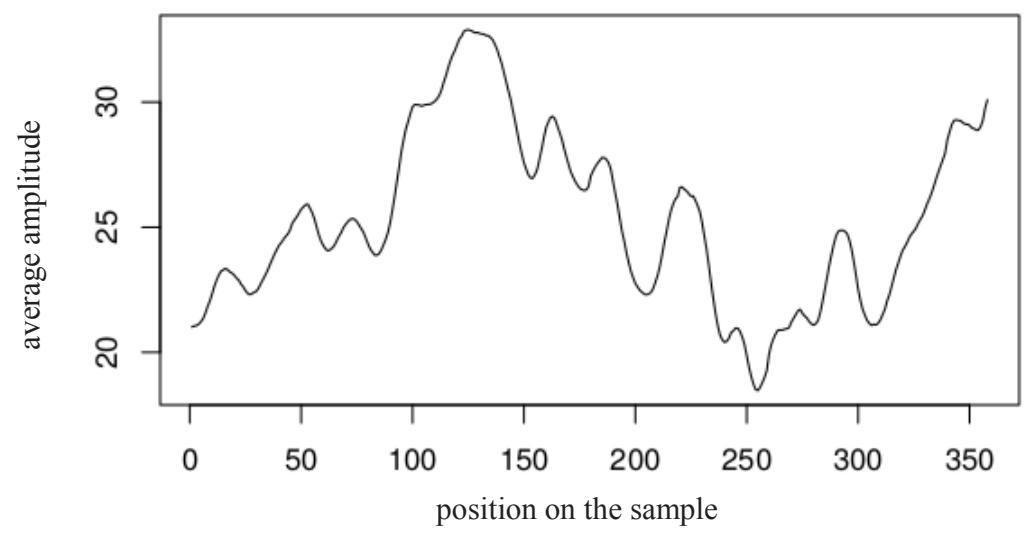

Fig. 6. The value of the time-averaged absolute value of the deflections of the sample at $300 \mathrm{~Hz}$

Also the time-averaged value of the absolute deflection at a given sample location was determined. The result from the example in figure 5 is shown in figure 6 . It explains that even for low frequencies, for which the vibration amplitudes of samples were largest, they did not exceed several dozen nanometres. The time-averaged amplitude allows to determine the position of the end of the metal element (see figure 7).
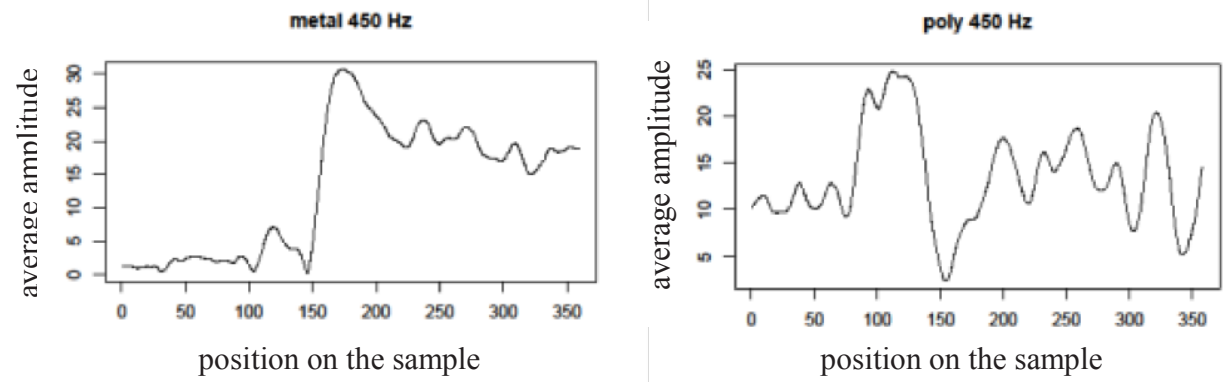

Fig. 7. Time-averaged value of the absolute deflections of the sample surface at $450 \mathrm{~Hz}$; observation on the side with the metal part on the left, on the full polymer side to the right; the metal part ranges from 150 to 360 and the piezotransducer from 0 to 20 .

We can detect the boundary between the polymer and the steel piece (in figure 7 at position 150). The difference between the deflections of the polymer and steel surface is few nanometres if the connection is functional. In the event of a connection failure, the difference in amplitudes is larger. 

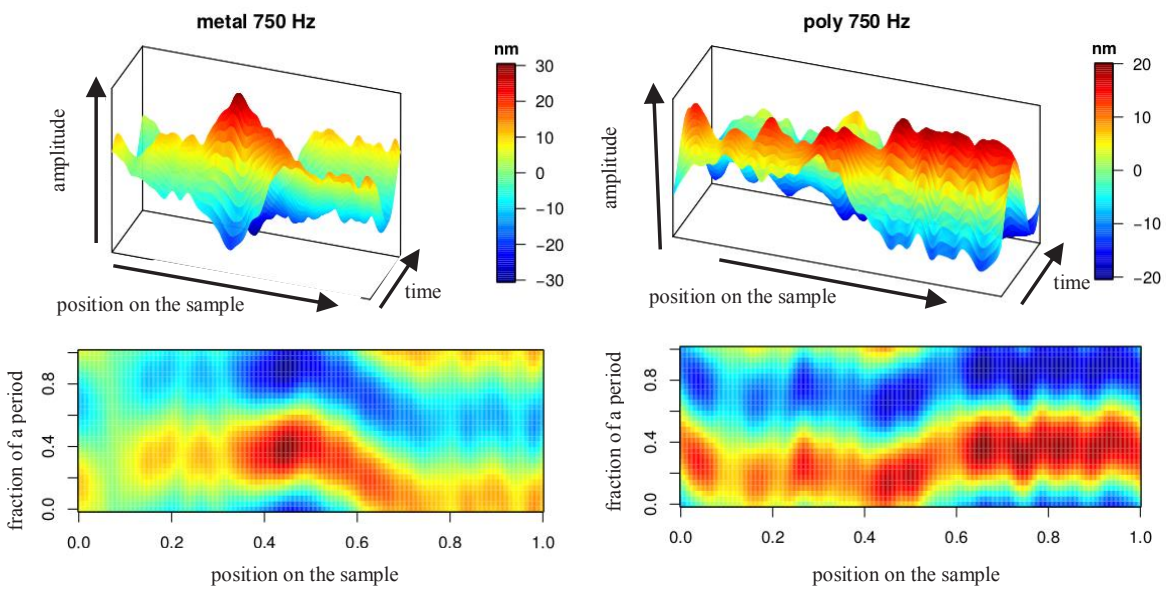

poly $750 \mathrm{~Hz}$
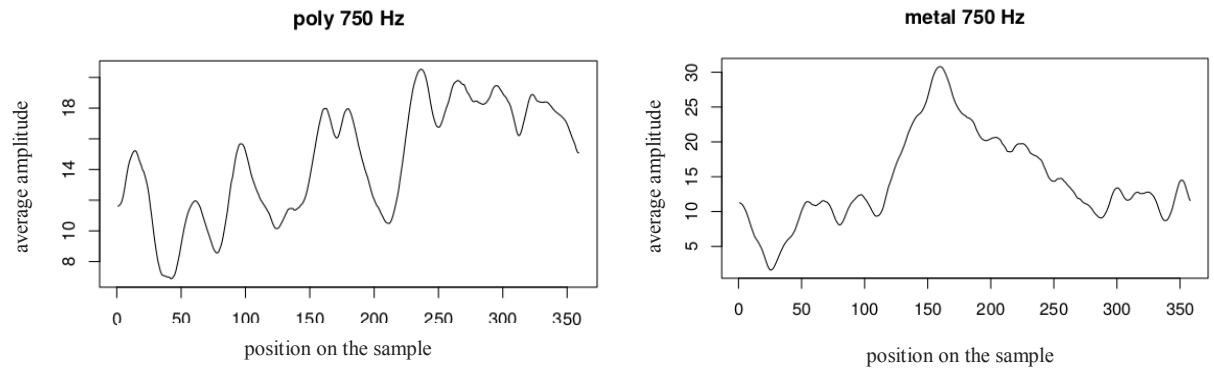

Fig. 8. Visualization of the deflection of the sample at $\mathrm{f}=750 \mathrm{~Hz}$ and the development in time of the deflections, observed from the side of the metal-polymer connection (left) and from the polymer side (right).

In the figure 8 the maximal average amplitude of vibrations on the part of the sample with only polymer on both sides is $20 \mathrm{~nm}$, while the vibrations of the metal are up to 1.5 times higher.
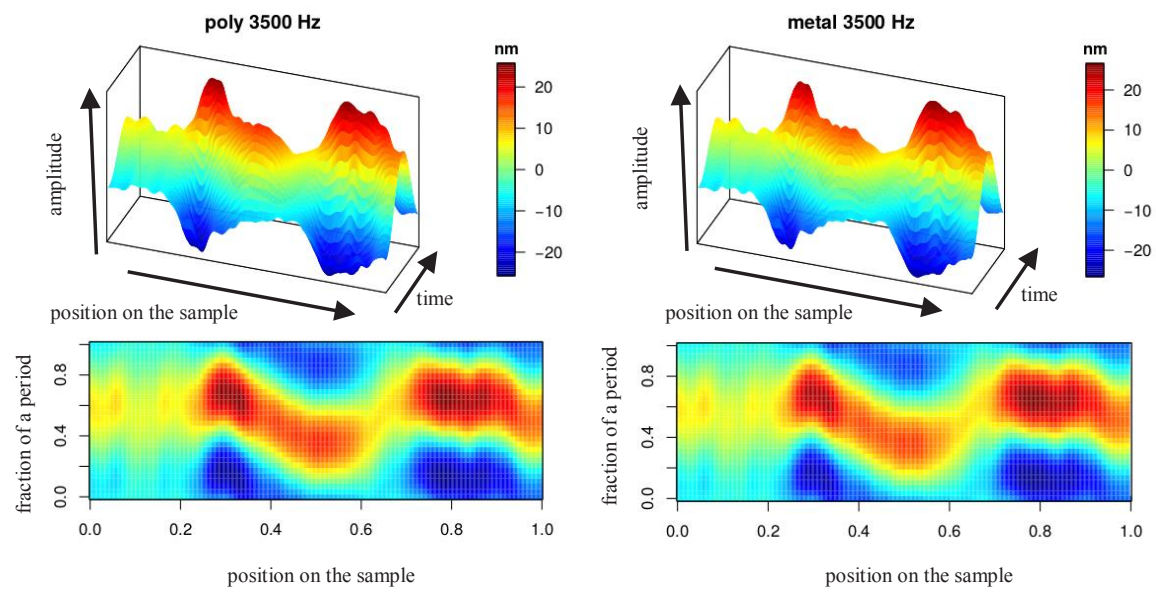

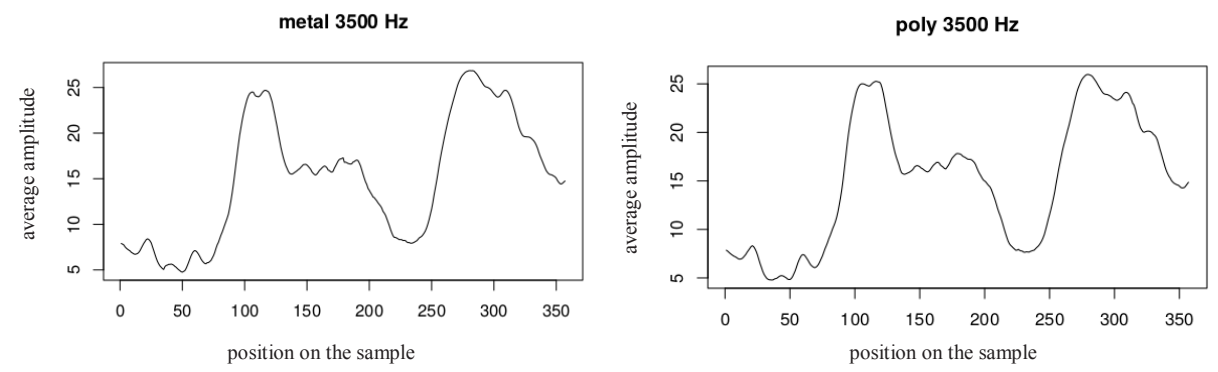

Fig. 9. Visualization of the deflections of the sample at a higher frequency ( $\mathrm{f}=3500 \mathrm{~Hz}$ ) and the time-averaged absolute value of the deflections, observed from the side of the metal-polymer connection (left) and from the polymer side (right).

In the figure 9 the maximal average amplitude of vibrations on both sides of the sample is $25 \mathrm{~nm}$, and the vibrations are consistent in terms of the value of deflection and phase. For each sample, the formation of standing waves with amplitudes from several to several tens of $\mathrm{nm}$ was seen. Higher vibration amplitudes were observed for lower frequencies (around $100 \mathrm{~Hz}$ ). The frequency at which a given standing wave is detected in the sample is determined with an accuracy of $10 \mathrm{~Hz}$. We observed that vibrations on the metal side had a larger amplitude than vibrations on the polymer side. At higher excitation frequencies (at least a few $\mathrm{kHz}$ ) vibrations on both sides were similar (see figure 9).

The quality of the polymer-metal connection can therefore be deduced by the similarity of the amplitude and phase of the vibrations observed on both sides: polymer and metal. In most cases, the vibration on the metal side has a much higher deflection amplitude compared with the pure polymer part. This resulted in vibrations with a larger amplitude in the part of the polymer connected to the metal. This creates the possibility of detecting a combination of a polymer-metal part in a situation where the observation of vibrations is possible only from the surface of the polymer. The results of time averaging over the absolute values of deflections, indicate particularly large values at the area of contact of the metal plate with the polymer. This allows to determine critical points in the structure connecting the polymer with the metal.

\section{Conclusions}

Comparison of vibration distributions and deflection values makes it possible to assess the quality of a polymer-metal connection. The proposed method of holographic vibrometry allows the diagnosis of the condition of this type of connections. During the measurements, none of the samples was damaged, and the analysis did not affect the quality of the connection, therefore it can be considered as non-destructive. Further research are required to obtain and present the main research results.

Acknowledgements: We thank K.Bula for preparation of the samples. ES acknowledges thanksfully partial support from grant $02 / 22 / \mathrm{SBAD} / 1501$ of the Poznan University of Technology 


\section{References}

1. P. Kah, R. Suoranta, S. Martikainen, C. Magnus, Rev. Adv. Mater. Sci. 36, 152-164 (2014)

2. J.W. Kaczmar, R. Wróblewski, L. Nakonieczny, J. Iwko, Polimery 53, 7-8 (2008)

3. M. Karny, report, Institute of aviation 244, 97-108 (2016)

4. N. Mitrović, Z. Ebersold, S. Dukić, B. Jordović, A. Peulić, Sci. Sinter. 44, 177-185 (2012)

5. I. G. Scott, C. M. Scala, NDT Int. 15, 75-86 (1982)

6. Y. Y. Hung, Opt. Eng. 21, 391-395 (1982)

7. P. Aryan, A. Kotousov, C. T. Ng, B. S. Cazzolato, Struct. Control HLTH. 24, 4 (2016)

8. D.A. Derusova, V.P. Vavilov, S. Sfarra, F. Sarasini, N. V. Druzhinin, Polym. Test. 68, 70-76 (2018)

9. Meijer F., Bula K., Kucharski D., Stachowska E., Non-destructive deformation measurements and defect testing of polymer structures, The 24th Annual World Forum on Advanced Materials, Poznan (2016)

10. M. Zorović, A. Čoki, J. Pest Sci., 88, 107-112 (2014)

11. The R-project for statistical computing, https://www.r-project.org/about.htm, (accessed 4 July 2019) 